\title{
Cyborgs céramiques
}

\section{Ceramic cyborgs}

\author{
Kimberley Harthoorn ${ }^{1}$ \\ ${ }^{1}$ historienne de l'art, kimberley.harthoorn@sevresciteceramique.fr
}

RÉSUMÉ. En partant de la construction théorique du cyborg proposée par Donna Haraway, le présent article propose de revoir les patients porteurs d'une prothèse en céramique biomédicale et les coraux bouturés sur des supports alumine poreuse comme des " cyborgs céramiques ", porteurs d'une combinaison intime entre le corps et la céramique. L'idée de faire corps avec la céramique n'est pas circonscrite à la pratique médicale. Elle peut s'entendre de différentes manières, et notamment avec beaucoup d'œuvres présentées dans Formes vivantes. Grâce aux œuvres de l'exposition, l'article explore les filiations possibles de la figure théorique du cyborg céramique, qui permet de mettre en lumière la multiplicité et le caractère politique des rapports entre corps et céramique.

ABSTRACT. From the starting point of the cyborg as a theoretical construction by philosopher Donna Haraway, this article aims at reframing patients with ceramic bone prosthetics and coral artificially grown on ceramic structures as " ceramic cyborgs ", showing a deep intrication between the body and ceramics. The idea of " faire corps " (body making) with ceramics is not just a medical reality. It can also lend itself to different understandings, as shown in the Formes vivantes ("Living forms ") exhibition. Thanks to several artworks showcased in this exhibition, the article explores the possible genealogy of the " ceramic cyborg " as a theoretical construction, which underlines the multiplicity and the political aspects of the relationship between body and ceramics.

MOTS-CLÉS. Céramique contemporaine, Donna Haraway, Biosémiotique.

KEYWORDS. Contemporary ceramics, Donna Haraway, Biosemiotics.

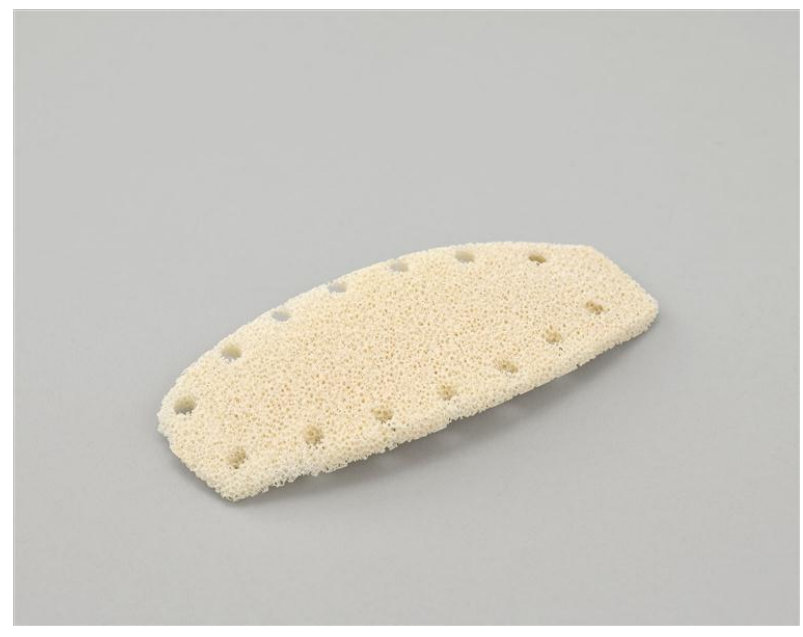

Sternum, I.CERAM, Limoges, 2015, Alumine poreuse, Limoges, musée national Adrien Dubouché , Don I.CERAM, 2016, ADL 13148. Photo (C) RMNGrand Palais (Limoges, musée national Adrien Dubouché) / Tony Querrec

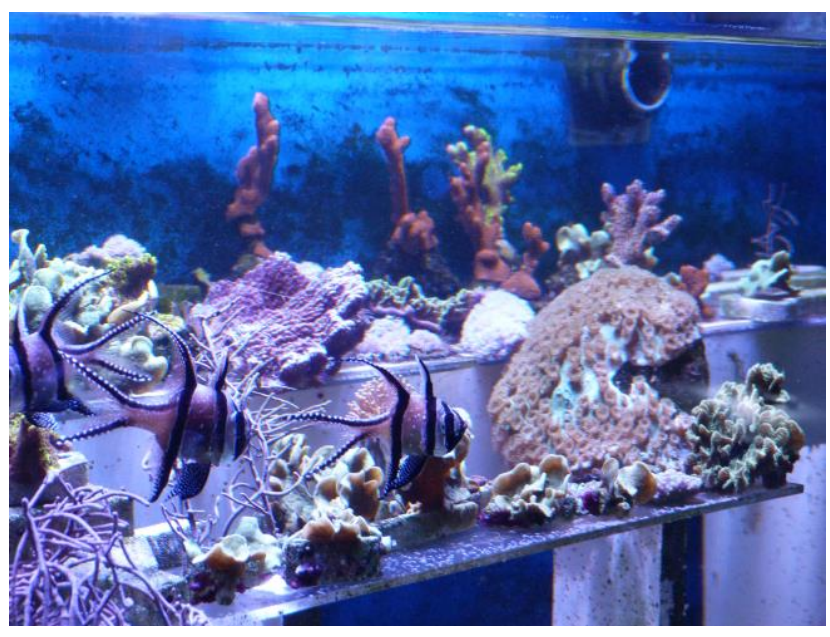

Aquarium du Limousin et I.CERAM, Bouture de corail sur alumine poreuse, Limoges, 2019, Limoges, aquarium du Limousin. Photo (O Musée national Adrien Dubouché.

Tout au long de Formes vivantes, projet d'exposition, de catalogue puis de colloque, un leitmotiv a rythmé les réflexions et guidé le choix des œuvres : le trouble de la frontière entre vivant et nonvivant. Au gré des exemples tirés de l'histoire, de la science et de l'art de la céramique, cette frontière a été déplacée, floutée, compliquée et multipliée. Les œuvres montrent des formes rampant de règne biologique en règne biologique, fleurissant et pourrissant, nous faisant voir leur peau et leurs nerfs, faisant croire qu'elles vivent et meurent comme des êtres. 
Cependant, à mon sens, le trouble a été le plus grand là où les frontières ont été repoussées le plus loin, c'est-à-dire hors du domaine de l'art, dans le monde concret de la biologie. Les prothèses en alumine poreuse présentées dans Formes vivantes ont la propriété de se combiner avec l'os humain d'une façon si intime que la prothèse et l'os guéri deviennent presque indiscernables. Le même matériau a été utilisé pour servir de support à des boutures de coraux réalisées par l'aquarium de Limoges. Dans les deux cas, l'organique et l'inorganique tissent des affinités au cœur de leur structure, dans leur matérialité même. Ce fait - que la céramique, matériau minéral, figé par le feu, se confond presque avec le vivant, concrètement - ouvre des perspectives nouvelles sur l'exposition dans son ensemble. C'est cette perspective que je souhaite explorer ici.

\section{Cyborgs céramiques}

Donna Haraway, philosophe, féministe, et historienne des sciences américaine, publie le Manifeste cyborg en $1985^{1}$. La figure du cyborg y est présentée comme le point de départ d'une critique de l'idée d'identité, comme une possibilité de subversion des frontières entre nature et culture, sujet et objet, soi et les autres. Selon Donna Haraway, les systèmes d'inégalités et de domination qui ont cours dans nos sociétés sont fondés sur ces limites, qu'il faut à tout prix subvertir. Ainsi, elle fait émerger le caractère politique des récits scientifiques, notamment lorsqu'ils s'occupent de distribuer les cartes ontologiques et de définir le réel auquel nous avons tous et toutes affaire.

Le Manifeste cyborg permet de concevoir tout corps comme composite, toute identité comme partielle et contradictoire. Le cyborg est une construction théorique qui se saisit de l'image du cyberorganisme informatique véhiculée par Terminator, Robocop et bien d'autres productions de la pop culture des années 80 à aujourd'hui. La figure, à la fois robotique et humaine, joue sur la peur de l'intégration du corps humain à un système technique aliénant. Haraway commente cette figure en rappelant que les corps féminins sont déjà modifiés par la médecine. Son travail permet ainsi de se saisir de cette figure du cyborg, de la faire nôtre : en effet, ce sont nos corps qui sont cyborg, faits de pièces rapportées, d'éléments biologiques étrangers, loin de l'idée que nous pourrions nous faire d'une « pure » humanité.

\section{Biosémiotique}

Le cyborg céramique n'est pas seulement une combinatoire de morceaux de corps dont certains seraient organiques et d'autres artificiels, mais comme une figure théorique, laboratoire d'affinités nouvelles entre les matières du corps et le matériau argile dans toutes ses potentialités, toutes ses mises en œuvre techniques. On peut alors proposer que l'idée du cyborg céramique est celle d'un « alliage » os-céramique, une figure qui cherche à nouer de nouvelles alliances matériologiques, non plus seulement entre métal et chair, mais aussi entre chair et céramique.

D'après Haraway, « aucun objet, espace ou corps n'est sacré en soi ; n'importe quelle composante peut être interfacée avec d'autres, si le bon code peut être construit afin de faire passer des signaux dans un langage commun ${ }^{2} »$. En somme, il faut une commune mesure pour pouvoir concevoir une alliance ou une affinité.

\footnotetext{
${ }^{1}$ Donna Haraway, Manifeste cyborg et autres essais, Sciences - Fictions - Féminismes, Anthologie établie par Laurence Allard, Delphine Gardey \& Nathalie Magnan, Paris, EXILS éditeur, 2007, 333 p.

${ }^{2}$ Donna Haraway, Manifeste Cyborg, Op. Cit. p. 32.
} 
Or, le corail bouturé et l'os fracturé qui se répare ont pour point commun de trouver dans l'alumine poreuse un matériau biocompatible. La céramique se substitue à l'os ou au support calcaire, structures du corps vivant. D'un point de vue que l'on pourrait appeler biosémiotique3, on assiste ici à l'énonciation d'un langage biologique commun entre humains et coraux, un élément qui est compris par les métabolismes des deux espèces. La céramique sert ici de support minéral métabolisable, pour les humains comme pour les coraux.

Nous partageons donc une situation avec les coraux : nous sommes réunis dans cette nécessité commune de nous structurer avec un matériau minéral, et dans cette capacité commune à s'allier au niveau biologique avec cette céramique, ce minéral artificiel. Il existe un langage commun, une condition commune qui nous permet de concevoir ces coraux, non simplement comme des éléments indispensables d'un écosystème planétaire en danger, mais aussi comme des êtres avec qui nous avons en commun au moins ça : une façon de faire corps avec le minéral, au niveau le plus intime.

Il faut prendre en considération les perspectives qu'ouvre cette potentialité du matériau céramique. Notre corporéité, notre dimension physique est constituée au niveau le plus intime d'alliances avec le non-humain. La prothèse céramique est une nouvelle modalité de cette alliance la catégorie du cyborg de Donna Haraway peut rendre compte de ce cas. Le corps humain est redéfini comme combinable avec une multitude d'autres éléments qui aliènent son identité et cassent l'idéal de la figure humaine.

\section{Comment élaborer des ontologies viables?}

Dans le Manifeste des espèces compagnes, Donna Haraway, revenant sur la question du cyborg, écrit ceci :

Mon objectif était d'investir les cyborgs selon une perspective critique, c'est-à-dire sans les célébrer ni les condamner, mais plutôt dans un esprit d'appropriation ironique, orienté vers des fins tout autres que celles imaginées par les guerriers de l'espace. A travers ce récit de cohabitation, de coévolution et de socialité interspécifique incarnée, le présent manifeste pose la question de savoir laquelle de ces deux figures, le cyborg ou l'espèce compagne, serait la plus à même de contribuer à l'élaboration de politiques et d'ontologies viables dans les mondes vécus contemporains. ${ }^{4}$

Ce texte, paru pour la première fois en 2003, soit bien après la première parution du Manifeste Cyborg, souligne l'enjeu de tels questionnements: «l'élaboration de politiques et d'ontologies viables dans les mondes contemporains ». Autrement dit, la question des alliances entre vivant et non-vivant, ou celle des affinités entre espèces, est porteuse d'une radicalité dans sa manière de déstabiliser les fondations ontologiques du monde tel qu'il est, et dans son pouvoir de faire signe vers de nouvelles ontologies plus viables.

Pour ce faire, Donna Haraway emploie deux outils par lesquels elle entend contribuer à de nouvelles ontologies: le récit et la figure. Elle met au jour leur potentiel critique, c'est-à-dire leur pouvoir de subvertir les grands récits hégémoniques et leurs effets pervers sur l'assignation des êtres vivants à des rôles immuables au sein de l'ordre social. En mobilisant le pouvoir transformateur de la fiction, on peut tenter de déplacer ces lignes de partage social et leurs fondations ontologiques.

\footnotetext{
${ }^{3}$ Champ d'étude à cheval entre biologie et étude du langage, la biosémiotique étudie les façons de faire signe et de communiquer au sein de la biosphère. La position de Donna Haraway s'apparente à cette nouvelle manière d'envisager le vivant avec les outils de la sémiotique, une perspective qui inclut le vivant dans le champ des sciences humaines.

${ }^{4}$ Donna Haraway, Manifeste des espèces compagnes, Paris, Flammarion, 2019, p. 26.
} 
Comme le fait remarquer Donna Haraway, la position du cyborg se trouve à la limite de différentes catégories ontologiques. Cette zone d'indistinction, de cristallisation entre catégories habituellement distinctes, cybernétique et organique, questionne la pertinence de ces catégories, de leur définition et de leurs conséquences sur l'imaginaire social.

L'élaboration et l'examen critique de figures telles que le cyborg permettent de peser le poids de la fiction dans notre perception du monde. Donna Haraway, insistant sur la nécessité d'effacer la ligne de partage entre nature et culture, permet au cyborg d'incarner cette réalité composite des corps qu'elle cherche à faire voir.

La forme vivante qu'à mon tour je cherche à faire voir, le cyborg céramique, est cette configuration liant intimement corps et céramique, brouillant la frontière entre vivant et inerte, ainsi que la frontière entre corps vivants. Cependant, je veux aussi montrer que cette figure, loin d'être un arrangement contemporain, connaît des précédents dans notre histoire culturelle. On va le voir, ces figures dont le cyborg céramique hérite ne mènent pas toujours à des ontologies viables.

\section{Faire corps avec la céramique}

Comment pouvons-nous «faire corps » avec la céramique ? Cette question semble sous-tendre l'exposition, elle la travaille de partout, par en-dessous. Certaines œuvres troublent les limites du corps, montrant que les liens entre céramique et corps humain peuvent se révéler intimes au point de se confondre, et pas seulement au niveau médical.

\section{Projections}

Dans l'introduction aux actes d'un colloque intitulé «Projections : des organes hors du corps ${ }^{5}$ », qui a eu lieu en 2006, Anne Simon et Hugues Marchal présentent des œuvres des domaines littéraire et artistique comme « une forme de trafic frontalier, assumé ou clandestin », entre corps et œuvre. Ils introduisent leur propos avec cette phrase de Merleau-Ponty, qui éclaire d'un regard différent les œuvres de Formes vivantes: «Où mettre la limite du corps et du monde, puisque le monde est chair 6 ?

\footnotetext{
${ }^{5}$ Anne Simon, Hugues Marchal dir., Projections : des organes hors du corps, actes de colloque, 13-14 octobre 2006, Musée d'Art contemporain du Val-de-Marne, Paris III-CNRS, en ligne, 2008, p. 2.

${ }^{6}$ Maurice Merleau-Ponty, Le visible et l'invisible, Paris, Gallimard, 1964, p. 182.
} 


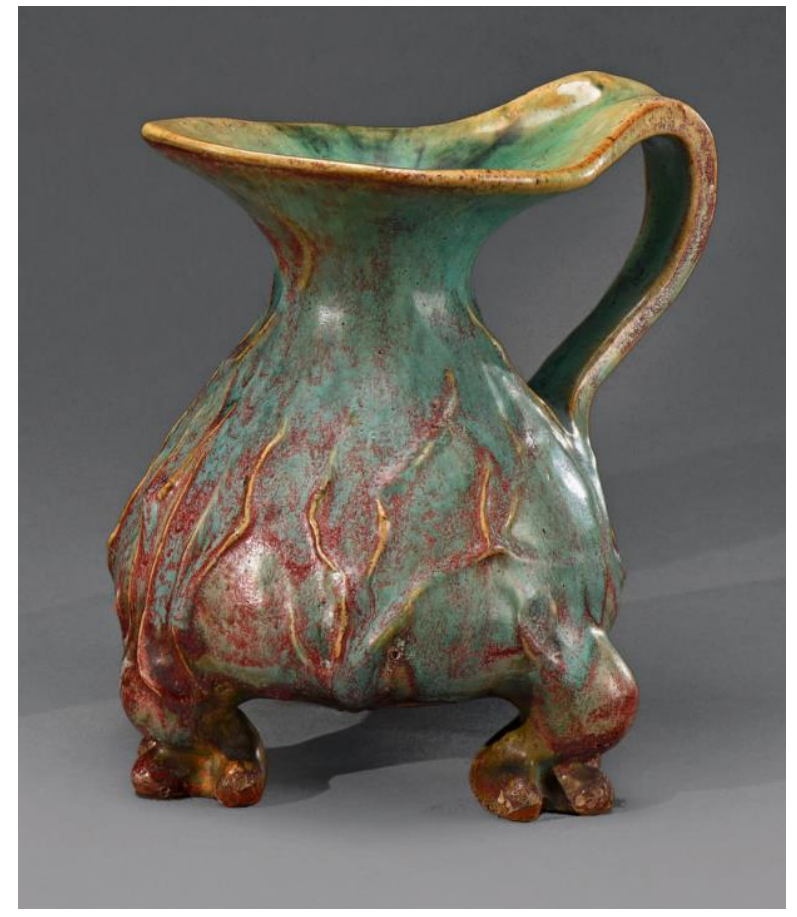

Pierre-Adrien Dalpayrat (1844-1910), céramiste, Alphonse Voisin-Delacroix (1857-1893), sculpteur, Vase Viscère, 1892-1893, Grès émaillé, hauteur: 25 cm, Lyon, collection Michel Laporte. Photo (C) De Baecque et Associés.

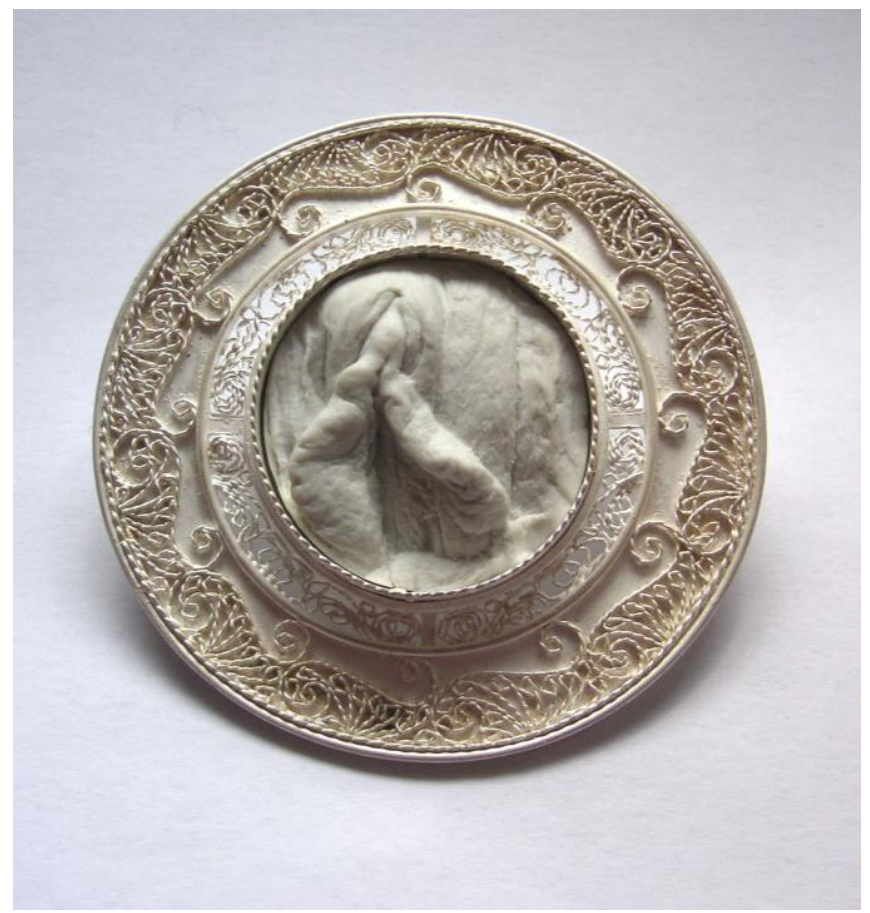

Carole Deltenre (née en 1983), Nymphes, 2008, Série de neuf broches, Porcelaine, argent, hauteur: $9 \mathrm{~cm}$, Collection de l'artiste, Photo (C) Carole Deltenre

Cette citation résonne particulièrement avec le vase-viscère de Pierre-Adrien Dalpayrat. Comme irrigué par un système sanguin, le vase fait allusion aux visions anthropomorphiques qui se cachent dans le vocabulaire descriptif de la céramique, comme la panse ou la lèvre. Simon et Marchal le rappellent : «Un certain nombre de thèses, en sciences humaines, identifient la production des artefacts humains à un phénomène de projection hors du corps de nos fonctions physiques et organiques, notamment les travaux importants d'anthropologues comme Lévy-Bruhl ou LeroyGourhan $^{7} »$. Le vase-viscère est comme un vase qui serait conscient de sa nature para-organique.

Cette logique de projection semble également animer l'œuvre Allô ${ }^{8}$ de Valérie Delarue. La sculpture, à l'émail riche de nuances, représente un cœur dont l'une des artères s'ouvre comme un pavillon. Ce cœur à l'écoute joue sur le double sens du mot cœur, à la fois organe et centre émotionnel. Le réalisme cru de la pièce laisse une impression de vulnérabilité totale. Vue au travers du prisme de la projection hors du corps de nos fonctions physiques, l'œuvre devient alors le réceptacle de notre sensibilité physique : une sculpture faite d'argile cuite qui pourtant nous semble à l'écoute.

L'une des spécificités du travail de l'argile et de la céramique est la possibilité de prendre des empreintes. Lors du transfert d'une forme «vivante » à l'argile, bras, jambe, visage, sexe, lézard, grenouille ou feuille, c'est le souvenir fantomatique d'une présence qui se maintient9. Dans les

\footnotetext{
${ }^{7}$ Anne Simon, Hugues Marchal dir., Projections : des organes hors du corps, op. cit., p. 3-4.

${ }^{8}$ Voir le catalogue de l'exposition Formes vivantes, Jean-Charles Hameau et Céline Paul (dir.), Milan, Silvana Editoriale, $2019, \mathrm{p}$. 207.
}

${ }^{9}$ Georges Didi-Huberman, La ressemblance par contact. Archéologie, anachronisme et modernité de l'empreinte, Éditions de Minuit, Paris, 2008. 
exemples montrés dans Formes vivantes, le masque mortuaire en porcelaine de William Guérin ou bien les vulves des Nymphes de l'artiste Carole Deltenre ${ }^{10}$, la notion de vivant contamine la céramique. Cette dernière se place à la limite du vivant, limite qui est ici une surface de contact, qui semble receler la présence du corps, et ce, malgré le retrait propre à la porcelaine.

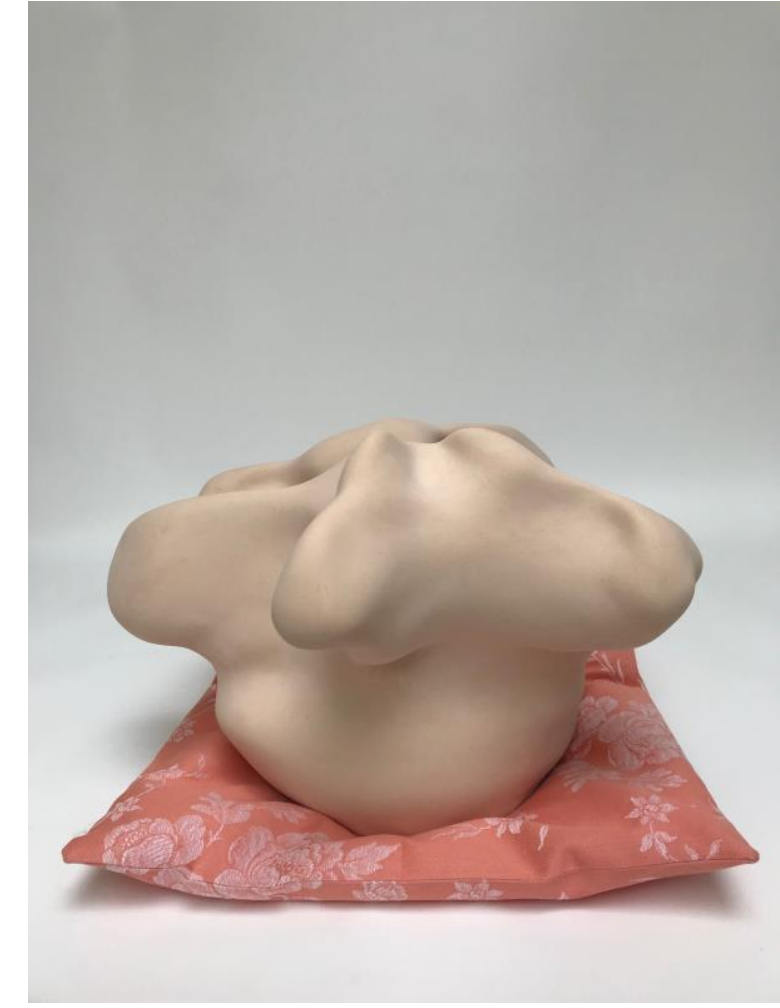

Farida Le Suavé (née en 1969), Little Bloom, 2019, Céramique et coutil fleuri, Collection de l'artiste / Courtesy Galerie Maria Lund, Photo Farida Le Suavé

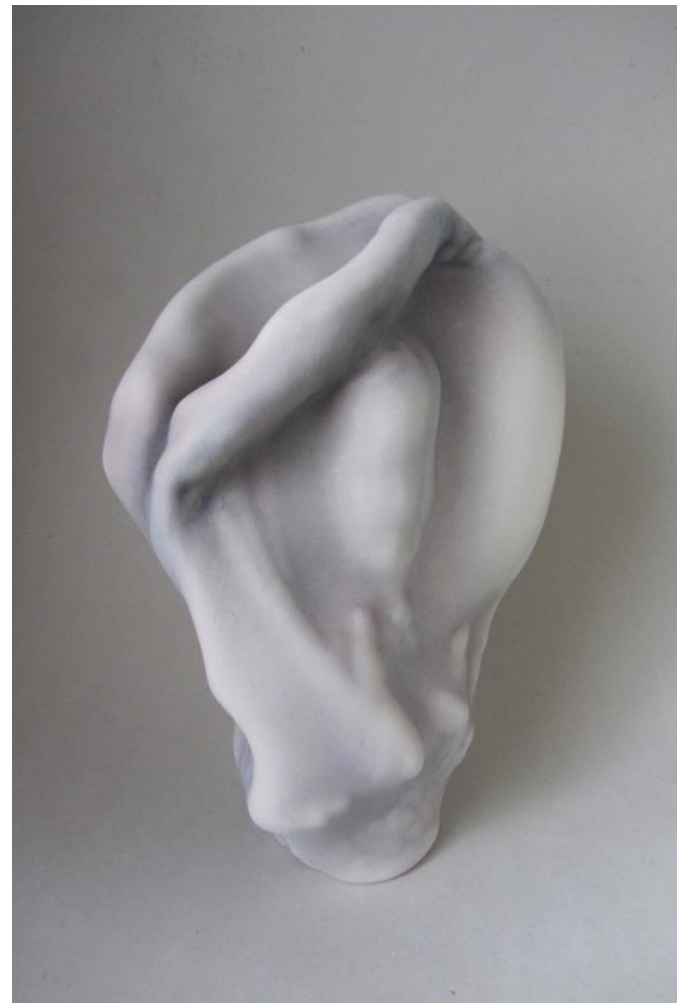

Wayne Fischer (né en 1953), 9032c, Porcelaine, 2018, Collection de l'artiste, Photo @ Wayne Fischer

\section{L'argile a-t-elle un genre?}

La question du genre se pose dans ces représentations céramiques. Les sculptures organiques de Farida Le Suavé et Wayne Fisher, que l'on pourrait voir comme abstraites, s'ouvrent comme des muqueuses, combinant l'aspect charnel des objets avec leur pénétrabilité. Les propriétés des techniques de la porcelaine et de la faïence sont mises en jeu pour faire varier les qualités de la chair, plastique chez Le Suavé, membrane chez Fisher. Ce lien entre céramique et pénétrabilité de la forme ouverte permet de réfléchir à l'idée, à première vue étrange, que l'argile et la céramique puissent être genrées.

On retrouve une forme de «féminisation» de l'argile, vue comme matériau passif et sensible, recevant forme, dans l'idée du schème hylémorphique. Gilbert Simondon s'est attaché à déconstruire ce schème $^{11}$ : selon lui, l'idée de matière passive, mise en forme par l'action de la technique, est une

\footnotetext{
${ }^{10}$ Voir le catalogue de l'exposition Formes vivantes, Jean-Charles Hameau et Céline Paul (dir.), Milan, Silvana Editoriale, 2019, p. 93 et 97.

${ }^{11}$ Estelle Zhong explique cet aspect de la pensée de Simondon, en rapprochant sa pensée de la "prise de forme" de celle de l'historien de l'art Henri Focillon. Estelle Zhong, "Des formes cachées dans la matière. La bricologie de l'art participatif à la lumière de la pensée de Gilbert Simondon". Techniques \& Cultures N64, 2015, en ligne, p. 4.
} 
vision fausse de la réalité du travail de la matière. En réalité, la matière contient un nombre limité de possibilités et de façons de travailler. En étant sensible à ce que la matière permet plus facilement, la mise en œuvre du matériau devient différente. En ce qui concerne l'argile, on sait par exemple que sa pratique doit composer avec la mémoire de forme inhérente à la structure du matériau. Le récit hylémorphique ne se vérifie pas : il s'agit bien d'une fiction.

On peut remarquer que cette fiction met en scène une opposition nette et tranchée entre passivité totale du matériau et principe actif de la mise en œuvre. Nous pourrions donner le nom de récit hylémorphique au récit qui fait du travail de la matière une relation entre principe passif et principe actif. Le récit d'une argile féminine et passive semble faire écho aux formes céramiques ouvertes, pénétrables, dotées d'une texture de chair. Ce récit hylémorphique s'établit sur le modèle d'une relation polarisée et hiérarchisée dont la structure est similaire à celle du récit de la binarité des genres. Il en épouse la netteté, la simplicité, et la violence conceptuelle.

L'idée de faire corps avec la céramique est très présente dans Formes vivantes. Que ce soit par la projection de fonctions du corps dans des formes céramiques, ou par la prise d'empreinte de corps humains dont les formes survivent ainsi dans le temps, la céramique se prête à un jeu trouble avec le monde organique. Elle agit comme interface entre le corps et le monde, comme lieu privilégié des trafics de (double) sens, des projections du corps dans le monde et du monde dans le corps.

Ces possibilités ainsi ouvertes, la question reste celle des ontologies viables : une fois les identités des corps et des œuvres rendues à leur complexité, remises en question depuis et par leurs limites, quels transferts de sens s'opèrent, et comment choisir ceux qui nous conviennent, qui nous permettent de vivre?

\section{Généalogies du cyborg céramique}

\section{Golems et robots}

Un transfert de sens s'établit alors, non entre une œuvre et une partie du corps, mais entre l'argile et l'idée de chair. Il y a transfert de substance : l'argile est faite chair, la chair faite argile. Ce transfert de sens ne serait pas aussi évident sans le souvenir du texte biblique. Dans la Genèse, le matériau dont Dieu se sert pour créer l'homme est bien l'argile. L'idée de chair-argile peut donc déjà être présente à l'esprit lorsqu'un visiteur regarde les œuvres.

Le lien de consubstantialité entre chair et argile est au cœur de la figure du golem. Selon le mythe, le rabbin Löw crée cette figure quasi humaine en argile et l'active par un signe écrit12. Cette figure du golem est multiple, comme en atteste le catalogue de l'exposition Golem qui a eu lieu au Musée d'art et d'histoire du judaïsme en 201713.

Le catalogue de l'exposition souligne la parenté entre la figure du golem et celle du robot. On y apprend que «dans l'imaginaire popularisé par la littérature et le cinéma de science-fiction, c'est le

\footnotetext{
${ }^{12}$ L'histoire se déroule à Prague, au XVIe siècle. Le rabbin Löw, qui habite dans le ghetto de Prague, décide de protéger sa communauté contre une attaque (selon les versions, un pogrom, une fausse accusation, etc), en fabriquant un colosse d'argile à figure vaguement humaine. Il est amené à la vie, soit par un morceau de papier glissé dans sa bouche avec le nom de Dieu écrit dessus, soit avec une inscription sur le front : EMET, vérité. Il peut agir, mais est dépourvu de parole. Le golem peut être désactivé, soit en retirant le papier de sa bouche, soit en effaçant la première lettre du mot, ce qui fait MET, mort.

${ }^{13}$ Ada Ackerman dir., Golem. Avatars d'une légende d'argile, catalogue d'exposition, du 8 mars au 16 juillet 2017, Musée d'art et d'histoire du judaïsme, Paris, Hazan, 2017.
} 
robot, doté d'une intelligence artificielle de plus en plus performante, qui incarne la figure du Golem. On sait que le substantif « robot», inventé par l'écrivain tchèque Josef Capek (1887-1945) (le terme « robota » signifiant en tchèque « labeur ») fut employé pour la première fois par son frère Karel Capek (1890-1938) dans sa pièce de théâtre R.U.R (Rossum's Universal Robots) écrite en 1920. Il y désigne les entités artificielles fabriquées par la science qui anéantissent l'humanité et viennent se substituer à elle14. »

Ces robots de théâtre « appartiennent à la même famille que le Golem, et, bien que fabriqués sur une île lointaine, plongent leurs racines dans l'humus, les maléfices de Prague. Le Golem est une image d'argile dotée de vie grâce au chem, le feuillet portant le nom de Dieu. De même, les robots ne sont pas des enchevêtrements de ressorts et de pistons comme les automates de foire, mais un conglomérat fait d'une substance chimique qui se comporte comme le protoplasme, d'une "gelée organique $^{15} \gg$ pour reprendre le terme de Josef Capek, substance découverte par le savant philosophe Rossum (rozum, c'est à dire raison), un «vieil extravagant», un « fou prodigieux » de la famille de ces fous savants nombreux dans l'expressionnisme ».

Le golem et le robot sont donc des figures apparentées. Ces figures issues de récits mythiques, souvent réécrits et surtout encore en activité dans la culture populaire, sont des opérateurs qui activent un transfert de sens entre chair et argile, qui le colorent, le connotent, d'une façon qui n'est pas sans poser problème.

En effet, si la notion de corps contamine la céramique en la rendant presque vivante, la notion d'argile, elle, contamine le corps en le rendant malléable, manipulable, pénétrable et contrôlable. C'est ce que suggèrent ces figures jumelles du golem et du robot : que la nature de nos corps serait d'être informés, modelés et contrôlés par des forces désirantes qui vont nous mettre au travail, que ce soit la volonté du rabbin Löw, ou celle du professeur Rossum, voire même celle de Dieu ${ }^{16}$.

\section{Olympia}

La figure double du golem et du robot est une représentation d'un corps subalterne sans volonté propre, mis au travail par des forces supérieures. Cette conception du désir animant la matière reprend le récit hylémorphique et sa distribution genrée des rôles passif et actif. D'ailleurs, parmi les premiers robots de la culture occidentale, on retrouve des occurrences genrées. On peut citer Villiers de l'Isle-Adam, L'Eve future (1886) - qui baptise son automate féminine l'andréïde - ou encore

Maria, le personnage de Metropolis, réalisé par Fritz Lang (1927). Ces occurrences montrent que la figure du robot, subalterne existant à la limite de l'humain et de l'inhumain, est dès le départ ancrée dans le récit ontologique de la binarité du genre et de sa hiérarchie.

\footnotetext{
${ }^{14}$ Id. p. $117-118$.

${ }^{15}$ Ibid. p. 118.

${ }^{16}$ Dans un essai présent dans le catalogue de Golem, Marc-Alain Ouaknin propose de « penser le désir comme terme générique pour désigner les forces de liaison au cœur du vivant ». Il se demande " si " golem » ne signifie pas précisément « matière animée de désir ». Golem, Op. cit. p. 144.
} 


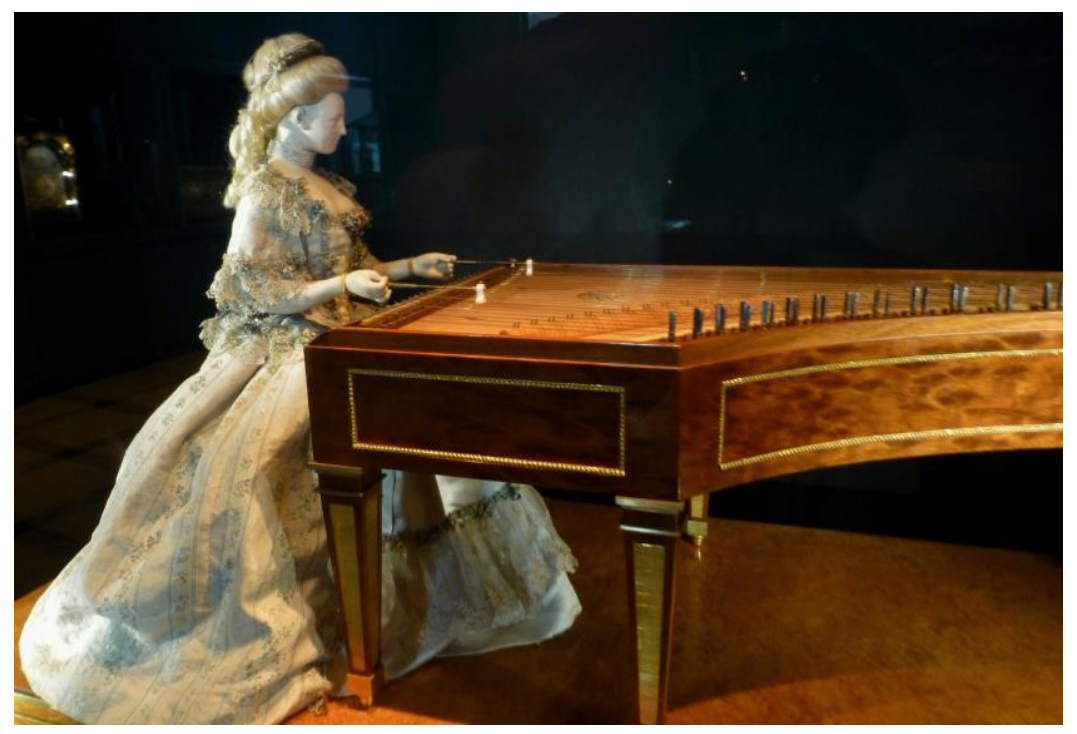

Pierre Kintzing, La joueuse de tympanon, 1784, divers matériaux dont soie, porcelaine, ivoire, acier, bois, laiton Paris, Musée des Arts et Métiers, 07501-0001. Photo OWikimedia commons

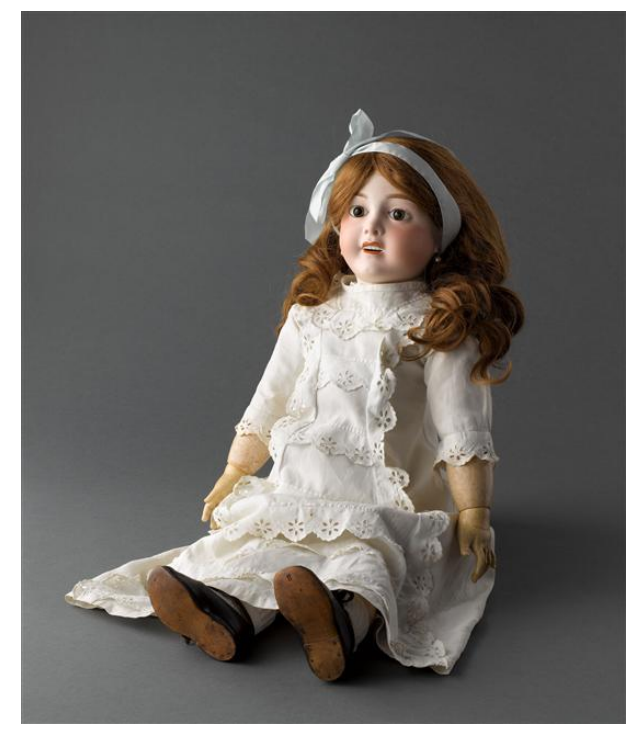

Poupée, modèle de E. Tasson, vers 1915-1920; carton pate, textile, porcelaine (manufacture Lanternier, Limoges), Musée national Adrien Dubouché, ADL 11784, Photo (C) RMN-Grand Palais (Limoges, musée national Adrien Dubouché) / Jean-Gilles Berizzi.

On dispose d'un exemple d'automate au musée des Arts et Métiers de Paris : la Joueuse de Tympanon17. Daté de la fin du XVIII ${ }^{\mathrm{e}}$ siècle, l'objet comprend un mécanisme qui actionne une figure féminine pour qu'elle joue d'un instrument de musique. L'artifice spectaculaire est conçu pour divertir et enchanter. L'automate est dotée d'un visage en porcelaine.

Le musée national Adrien Dubouché conserve également plusieurs poupées dont les têtes ont réalisées en porcelaine de Limoges ${ }^{18}$. La composition des têtes de poupée fait de la céramique un matériau incarnant un idéal physique, une certaine qualité de chair rendue par la porcelaine :

\footnotetext{
${ }^{17}$ Joueuse de tympanon, Pierre Kintzig, divers matériaux dont soie, porcelaine, ivoire, acier, bois, laiton, 1784, Musée des Arts et Métiers, Paris.

${ }^{18}$ Le musée national Adrien Dubouché en conserve quelques-unes, comme par exemple la Tête de poupée, manufacture Lanternier, biscuit de porcelaine, 1917, Musée National Adrien Dubouché, Limoges.
} 
blanche, pure, douce, homogène, luxueuse et fragile. Vinciane Despret, dans sa préface au Manifeste des espèces compagnes, fait la remarque suivante : "Quand Haraway évoque la "pure race » de berger australien Cayenne ou le "mélangé » Roland, elle sait ce que ces discours tissent de complicités avec les discours raciaux ${ }^{19}$. $\gg$ De la même manière, ici a lieu un tissage d'affinités avec un système de valeurs raciste et sexiste, d'une part par l'exaltation de la blancheur du matériau, et d'autre part par la valeur symbolique des objets coloniaux, dont la porcelaine est un élément important.

Cette joueuse de tympanon fait penser à l'automate Olympia, personnage de «l'Homme au sable », paru en 1816 dans les Contes nocturnes d'E.T.A Hoffmann, dont le personnage principal, l'étudiant Nathanaël, tombe amoureux sans s'apercevoir qu'elle n'est pas humaine. À la fin du conte, Hoffmann raconte avec ironie comment les gens de la ville réagissent lorsqu'ils réalisent qu' Olympia est un robot :

«L'histoire de l'automate avait jeté de profondes racines dans leur âme, et il se glissa en eux une affreuse méfiance envers les figures humaines. Beaucoup d'amants, afin d'être bien convaincus qu'ils n'étaient pas épris d'une automate, exigèrent que leurs maîtresses dansassent hors de mesure, et chantassent un peu faux ; ils voulurent qu'elles se missent à tricoter lorsqu'ils leur faisaient la lecture, et avant toutes choses, il exigèrent d'elles qu'elles parlassent quelquefois réellement, c'est-à-dire, que leurs paroles exprimassent quelquefois des sentiments et des pensées, ce qui fit rompre la plupart des liaisons amoureuses. »

Hoffmann montre comment ces rapports entre hommes et femmes se hiérarchisent, le désir des hommes se projetant vers des « objets » d'amour. Le récit de la binarité-hiérarchie des genres est ici commenté avec ironie : les hommes du récit ne désirent pas des femmes mais des automates.

Thierry Dufrêne, dans La poupée sublimée $e^{20}$, explique que l'essai de Freud intitulé «l'inquiétante étrangeté », paru en 1919, se fonde entre autres sur la lecture de «L'homme au sable » par Ernst Jentsch en 1906. Jentsch cite Olympia comme exemple pour définir l'inquiétante étrangeté : c'est l'incertitude concernant le fait qu'un personnage soit vivant ou non. Cette notion connaît une variation en robotique contemporaine : la «vallée de l'étrange », théorisée par Mori Masahiro. Selon lui, plus un robot ressemble à un humain, plus il semble monstrueux.

Hans Bellmer, en 1932, aurait eu l'idée de concevoir sa poupée après une représentation des Contes d'Hoffmann de Jacques Offenbach. Les poupées de Bellmer se placent directement dans l'héritage d'Olympia, figure d'automate sur laquelle se cristallisent de nombreux fantasmes. Elles mettent à nu la violence destinée aux corps assignés comme passifs dans la distribution ontologique des rôles de genre.

Thierry Dufrêne place Cindy Sherman comme héritière de Bellmer dans l'usage des poupées sexuelles, avec les broken dolls. "L'artiste insiste presque lourdement sur le simulacre - les faux nez, les faux nichons -, qui lui permet de déconstruire les stéréotypes des contes de fées pour petites filles sages. Avons-nous d'ailleurs une identité qui ne soit un jeu de dupes? Nous non plus, nous n'avons pas choisi notre rôle : on nous a imposé ce corps normé, ces occupations forcées. Nous aussi sommes factices, en plastique, comme les simulacres que nous voyons. Composés. ${ }^{21}{ }^{2}$

\footnotetext{
${ }^{19}$ Donna Haraway, Manifeste des espèces compagnes, Op. cit., p. 14.

${ }^{20}$ Thierry Dufrêne, La poupée sublimée : quand Niki de Saint-Phalle et les artistes contemporains font des poupées, Skira, Paris, 2014, p. 78.

${ }^{21}$ Id. p. 84. 


\section{Conclusion}

La figure multiple du cyborg est une figure du passage des frontières, c'est-à-dire de leur subversion et de leur complication. Thierry Dufrêne le dit bien : il y a un trouble dans l'identité, à voir ces poupées dont finalement on partage le destin. Ces figures, cyborgs, automates, golems, robots, continuent à peupler notre monde. Le cyborg genré est un corps fragmentaire, à l'identité partiellement humaine. Le type de corps qui est ainsi fabriqué est un automate manipulable, une poupée objet de désir, version genrée du golem. Le décrire, c'est essayer de rendre compte de la complexité, de la densité des réseaux de sens dans lesquels nous sommes pris.

La proximité entre chair et argile nous amène sur un terrain glissant, celui d'une condition humaine par essence condamnée à servir. Le destin d'Olympia ou du Golem ne semblent pas des ontologies viables pour l'être humain. Cependant, en esquissant l'histoire de ces réseaux de sens, on peut prendre conscience de leurs effets, et potentiellement les subvertir. On peut aussi s'émerveiller de leur complexité, et de toutes les possibilités ontologiques qu'ils offrent.

En effet, il y a des «trafics de sens ${ }^{22}$ » entre corps et céramique partout dans l'exposition. On a trouvé de la céramique dans l'organique, avec les coraux et les humains modifiés par les prothèses de la société ICÉRAM, et de l'organique dans la céramique, par le biais des viscères. Il y a du « aété-vivant » dans la porcelaine et dans le grès, avec les masques mortuaires. On a aussi trouvé de la chair dans l'argile et de l'argile dans la chair, à tel point qu'on finit par se demander si ce n'est pas le fait d'être quasi-humain ou moins-qu'humain qui est paradoxalement la marque de notre humanité.

Ainsi, l'idée d'affinité entre corps et céramique nous amène très concrètement à la possibilité de réparer des corps abîmés, coralliens ou humains, de nous découvrir des choses en commun avec d'autres espèces de la biosphère, animales ou végétales, mais aussi avec d'autres entités sur le spectre du quasi-humain, avec les cyborgs, les golems, les fantômes, les automates, les robots, les poupées ; et, peut-être, grâce à des opérateurs de toutes sortes, comme a pu l'être la céramique dans notre exemple, mais comme pourraient l'être d'autres choses encore à découvrir, pourra-t-on échafauder de nouvelles alliances, de nouvelles façons de reconstruire le monde, ou a minima des façon de l'habiter, qui soient « viables dans les mondes vécus contemporains ${ }^{23} »$.

\footnotetext{
${ }^{22}$ La notion est d'Anne Simon. Anne Simon, Hugues Marchal dir. Projections, Op. cit., p. 2.

${ }^{23}$ Donna Haraway, Manifeste des espèces compagnes, op. cit., p. 26
} 